\title{
Factores socioeconómicos que influyen en la inadecuada gestión integral de residuos sólidos en el distrito
} de María

\section{Socioeconomic factors that influence in the inadequate integral management of solid waste in the Maria district}

\author{
Sulamita Vargas Inga y Manuel Oliva ${ }^{1 *}$
}

\section{RESUMEN}

La presente investigación tuvo como objetivo determinar los factores socioeconómicos que influyen en la inadecuada gestión integral de los residuos sólidos en el distrito María. Los resultados explicaron que dentro del factor social, la edad, género, rol que cumple en la familia, participación en capacitaciones sobre residuos sólidos, grado de instrucción, integrantes de la familia, falta de participación ciudadana, escasa información, desconocimiento sobre la gestión integral de residuos sólidos y la educación ambiental, son los principales factores que influyen en la inadecuada GIRS (Gestión Integral de Residuos Sólidos). Sin embargo dentro de los factores económicos el número de personas que trabajan en el hogar, el ingreso promedio de la familia, y los escasos recursos económicos con los que cuenta la municipalidad, son en este caso los principales factores que influyen en esta inadecuada GIRS. Finalmente los resultados de la presente investigación permitieron realizar una propuesta para el mejoramiento de la GIRS en el distrito de María, teniendo en cuenta todos los factores antes tratados, tanto socioeconómicos como políticos.

Palabras clave: Factores socioeconómicos, gestión integral de residuos sólidos, manejo de residuos sólidos

\begin{abstract}
The present research had as goal to determine the socioeconomic factors that influence in the inadequate integral management of solid waste in the Maria district. The results explained that within the social factor, age, gender, family role, participation in trainings on solid waste, educational level, family members, lack of citizen participation, lack of information, lack of knowledge about comprehensive management solid waste and environmental education are the main factors influencing in the inadequate GIRS (Integrated Management of Solid Waste). However, within the economic factors, the number of people working in the household, the average income of the family, and the scarce economic resources available to the municipality are, in this case, the main factors that influence this inadequate GIRS. Finally, the results of the present research allowed to make a proposal for the improvement of the GIRS in the district of Maria, taking into account all the previously discussed factors, both socioeconomic and political.
\end{abstract}

Keywords: Socioeconomic factors, solid waste control, solid waste management

\footnotetext{
${ }^{1}$ Universidad Nacional Toribio Rodríguez de Mendoza de Amazonas (UNTRM-A), Instituto de Investigación para el Desarrollo Sustentable de Ceja de Selva, Calle Higos Urco N 342-350-356, Calle Universitaria N 304, Chachapoyas, Perú

a-mail:singa@indes-ces.edu.pe

*Autor de correspondencia: E-mail: soliva@indes-ces.edu.pe
} 


\section{INTRODUCCIÓN}

Durante muchos años, el hombre a través de sus prácticas diarias de tipo doméstico, comercial e industrial ha requerido procesos sencillos y complejos que han generado una diversidad de productos y residuos al ambiente. Esta generación de residuos sólidos ha tenido y tiene repercusión en el ambiente y en la salud de las personas. El problema no radica solo en la generación de los residuos ya que toda utilización de bienes genera desechos, la problemática repercute en la gestión integral de los residuos sólidos que incluye manejar las tareas con alto nivel de complejidad como en el transporte y disposición final de los mismos (Martínez, 2015).

Jaramillo (1999), observa que los problemas asociados a la gestión de los residuos sólidos en la sociedad actual son complejos, por la cantidad y variedad de residuos, por el desarrollo de las zonas urbanas dispersas, por las limitaciones de fondos para los servicios públicos en muchas ciudades, por los impactos de la tecnología, por las limitaciones emergentes de energía y materias primas y especialmente por la escasa o nula responsabilidad frente al problema de gestión, disposición y los costos asociados. Todas estas causas son debidas a la falta de algunos factores derivados de la educación ambiental y reconocimiento de reales posibilidades. Asimismo, menciona que la jerarquía de la gestión integral de residuos sólidos debe hacerse cumplir de una forma que sea suficientemente flexible como para permitir a los gobiernos municipales que implanten instalaciones de gestión de residuos sólidos acorde con las condiciones locales y la capacidad de pago de los usuarios con el fin de mejorar la gestión. Postand y Baud (2004), indican que la gestión integral de los residuos sólidos toma en cuenta a los actores que participan en ella, a los propios sistemas de manejo de desechos (recolección, tratamiento, disposición final), y las condiciones locales desde donde se implementa el sistema, incluyendo los aspectos técnicos apropiados, y la situación ambiental, financiera, sociocultural, institucional y política para diseñar y articular soluciones eficientes, efectivas e igualitarias. Se reconoce la dificultad al implementar dicho enfoque, pero en diversos trabajos se afirma que es la propuesta más adecuada y exhaustiva para concretar un correcto manejo de los desechos.

Por estas razones, en el presente estudio se pretende evaluar los factores socioeconómicos que influyen en la gestión integral de residuos sólidos en el distrito de María, en la reg

\section{MATERIALY MÉTODOS}

Área de estudio

El estudio se localizó en el distrito de María, situado en el ecosistema de los bosques pluviales montanos de la provincia de Luya, al suroeste del Departamento de Amazonas (Figura 1).

\section{Etapa preliminar}

Para determinar los principales factores socioeconómicos que influyen en la inadecuada Gestión Integral de Residuos Sólidos (GIRS) en el distrito de María, se trabajó con el muestreo aleatorio estratificado obteniendo de esta manera el tamaño de muestra en estudio, de la manera señalada en la tabla 1.

Tabla 1. Muestra aleatoria estratificada para una varianza especificada con población finita y afijación proporcional

\begin{tabular}{cll}
\hline & & $\begin{array}{l}\text { Proporción de la población } \\
\text { que evidencia un manejo } \\
\text { adecuado de los residuos } \\
\text { sólidos }\end{array}$ \\
\hline $1-\mathrm{p}=\quad 0,43 \quad 0,57 \quad \begin{array}{l}\text { Proporción de la población } \\
\text { que evidencia un manejo } \\
\text { inadecuado de los residuos } \\
\text { sólidos }\end{array}$ \\
\hline $\mathrm{E}=0, \begin{array}{l}\text { Precisión - máximo error de } \\
\text { estimación }\end{array}$ \\
\hline $\mathrm{z}=0 \begin{array}{l}\text { Valor de la distribución } \\
\text { normal para un nivel de } \\
\text { confianza del 93\% }\end{array}$ \\
\hline $\mathrm{V}=0,03626943 \begin{array}{l}\text { Varianza deseada } \\
\text { especificada }\end{array}$ \\
\hline
\end{tabular}

Varianza deseada o especificada

$$
\mathbf{V}=\frac{\mathbf{E}}{\mathbf{Z}}
$$




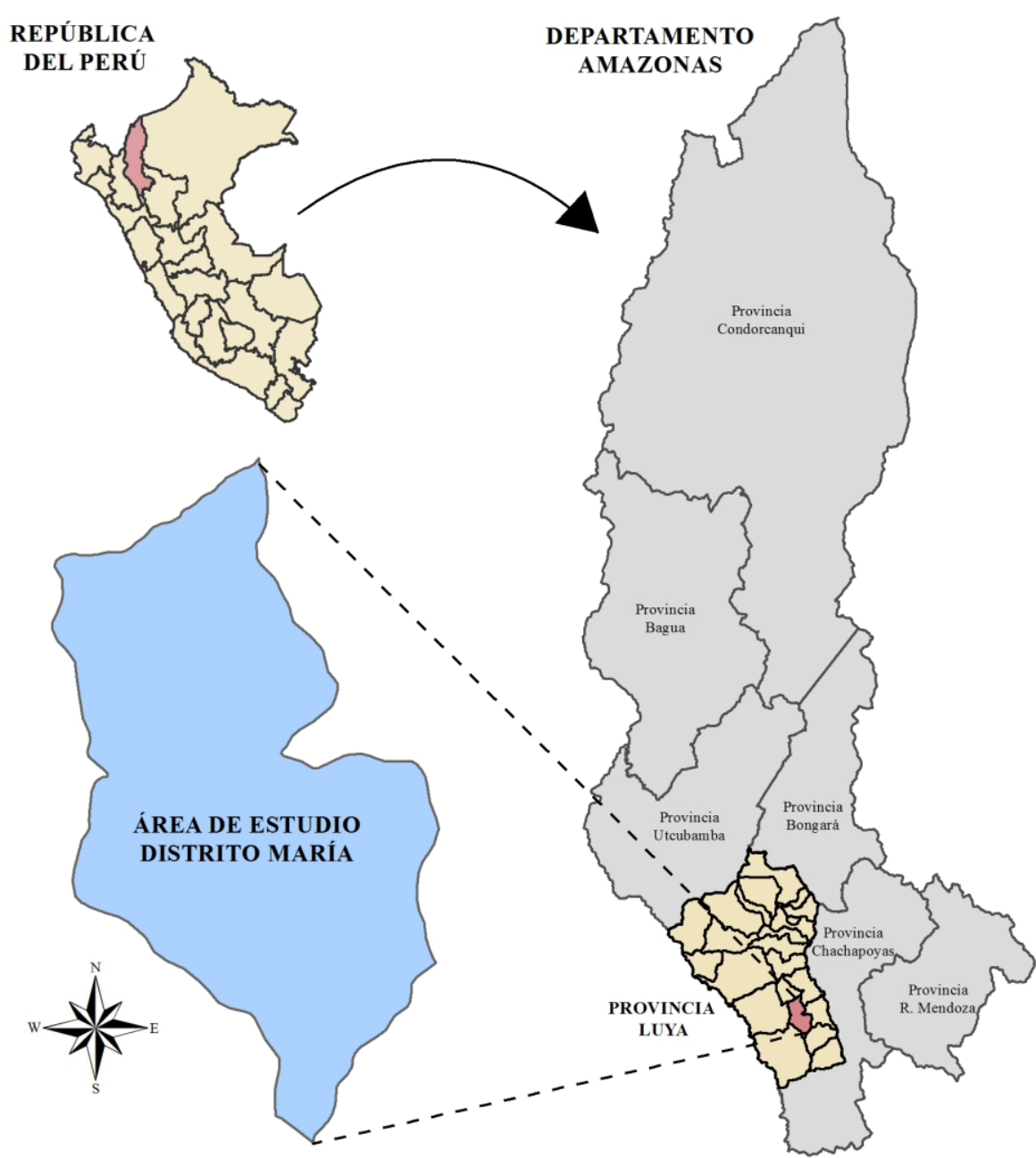

Figura 1. Ubicación de la zona de estudio en el distrito de María (provincia de Luya)

Tamaño de la muestra

$$
\mathbf{n}_{\mathbf{0}}=\frac{\sum \mathbf{W}_{h} \mathbf{P}_{h} \mathbf{Q}_{h}}{\mathbf{V}}
$$

$\mathrm{n}=150$

\section{Etapa de campo}

Durante esta etapa se realizó la aplicación de encuestas y fichas de observación a la muestra encontrada (Tabla 2).

Tabla 2. Distribución del tamaño de la muestra, según su tamaño poblacional

\begin{tabular}{|c|c|c|c|}
\hline Distrito maría & \multicolumn{2}{|c|}{ Población } & Muestra \\
\hline \multirow[t]{2}{*}{ Urbano } & María & 250 & 82 \\
\hline & Cuchapampa & 70 & 23 \\
\hline \multirow[t]{2}{*}{ Rural } & Quizango & 97 & 32 \\
\hline & Mangalpa & 39 & 13 \\
\hline \multicolumn{2}{|c|}{ Total } & 456 & 150 \\
\hline
\end{tabular}

\section{Etapa de procesamiento de datos}

En esta etapa se realizó el análisis descriptivo y la correlación lineal entre la variable dependiente y la independiente bajo el siguiente esquema:

$$
Y_{t}=\beta_{1}+\beta_{2} X_{t}+U_{t}
$$

donde:

$\mathrm{Y}_{\mathrm{t}}=$ Variable dependiente

$\mathrm{X}_{\mathrm{t}}=$ Variable independiente

$\mathrm{U}_{\mathrm{t}}=$ Perturbación aleatoria

$\mathrm{t}=1,2,3 \ldots, \mathrm{T}$

$\mathrm{T}=$ Observaciones de la variable $\mathrm{Y}, \mathrm{X}$ 


\section{RESULTADOS}

En cuanto al diagnóstico de la Gestión Integral de Residuos Sólidos se encontró que el $40 \%$ de la población no conoce la GIRS y el $60 \%$ si conoce la GIRS, tal como se muestra la figura 2.

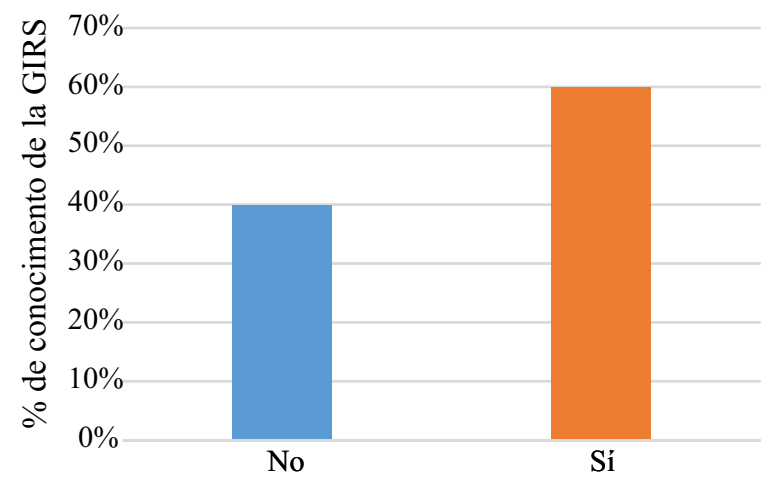

Figura 2. Población que conoce la GIRS.

En la evaluación y análisis de la Gestión Integral de Residuos Sólidos (GIRS), se encontró que no siempre los que conocen la GIRS hacen un manejo adecuado de los residuos sólidos. La figura 3, dio a conocer que el $100 \%$ de la población que si conoce la GIRS ha participado en cursos, talleres, campañas de sensibilización sobre residuos sólidos, sin embargo el $100 \%$ del grupo que no conoce la GIRS respondieron lo contrario.

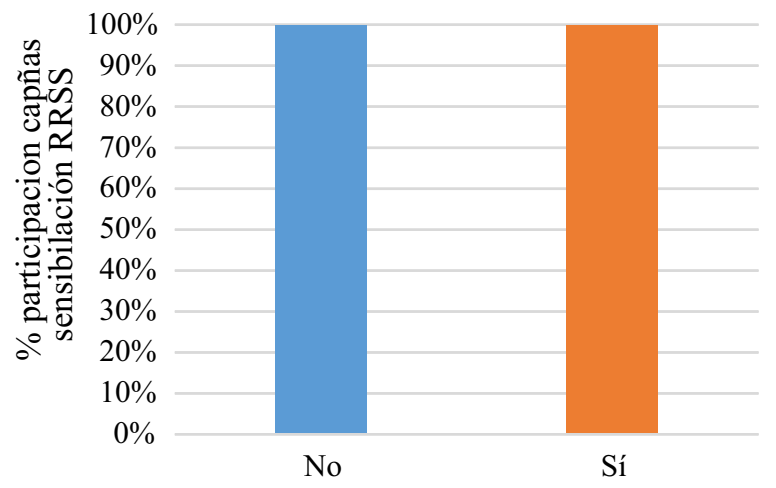

Figura 3. Capacitación en manejo de residuos sólidos (RRSS).

La figura 4 dio a conocer los motivos por los cuales no recibe el servicio de recolección. En este sentido, el mayor porcentaje que representa el $72,9 \%$ de los que conocen la GIRS, mencionó por no estar inscrito, mientras que el $71,9 \%$ de los que no conocen GIRS mencionaron lo mismo; sin embargo el $4,2 \%$ de los que conocen la GIRS y el $2 \%$ de los que no conocen la GIRS respondieron que son otros los motivos por el cual no reciben el servicio, tales como: la falta de personal, ya que solo se contó con uno y no se abastecían en recorrer todo el distrito; los costos son muy altos y poca eficiencia en el servicio; falta de información a la sociedad de los servicios que viene brindando la municipalidad; escasa participación de la población en el servicio que la municipalidad brinda.

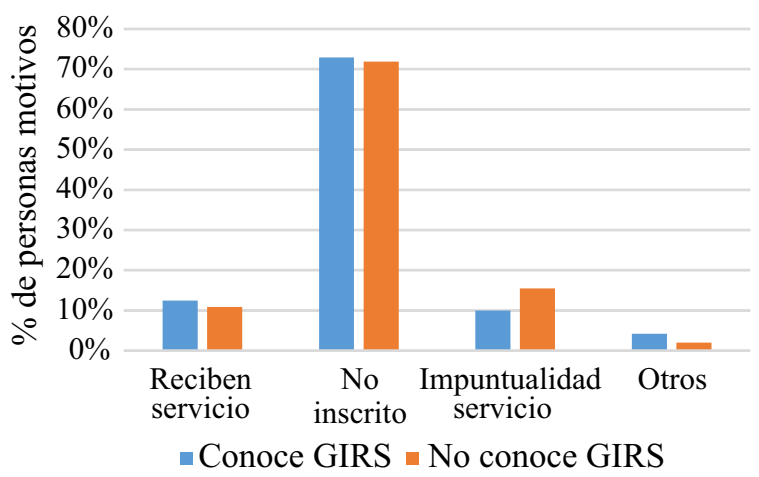

Figura 4. Motivos para no recibir el servicio de recolección de GIRS.

La figura 5 dio a conocer los motivos por los cuales no recibe el programa de segregación en la fuente. En este sentido, el mayor porcentaje que representa el 47,9\% de los que conocen la GIRS y el $47 \%$ de los que no conocen la GIRS, respondieron que son otros los motivos tales como: falta de asesoramiento a las autoridades en temas de residuos sólidos; no contaban con una area ambiental encargada de estos temas; e inadecuada distribución del FONCOMUN (Fondo de compensación municipal).

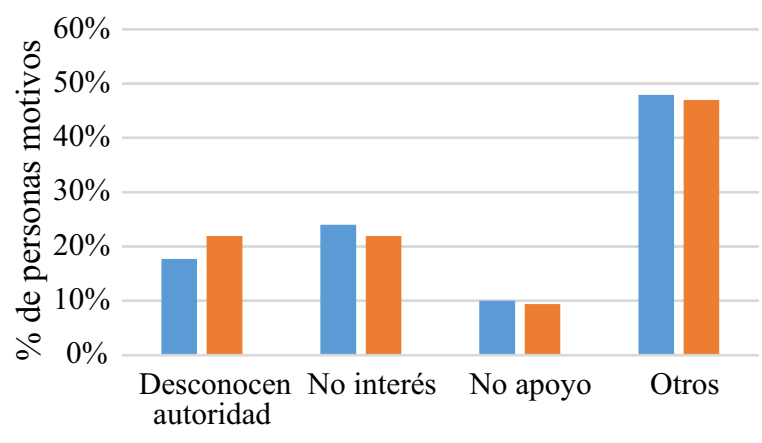

- Conoce GIRS - No conoce GIRS

Figura 5. Programa segregación en la fuente.

La figura 6 por su parte dio a conocer el lugar donde disponen finalmente los residuos sólidos. De esta 
manera, el mayor porcentaje, que representa el 90,6\% de los que conocen la GIRS y el $84,4 \%$ de los que no conocen la GIRS, mencionaron que el lugar es un vertedero o botadero de basura, seguido de al borde de la carretera ( 8 y $14,1 \%$, respectivamente) y del río (1,4\% y $1,6 \%$, respectivamente).

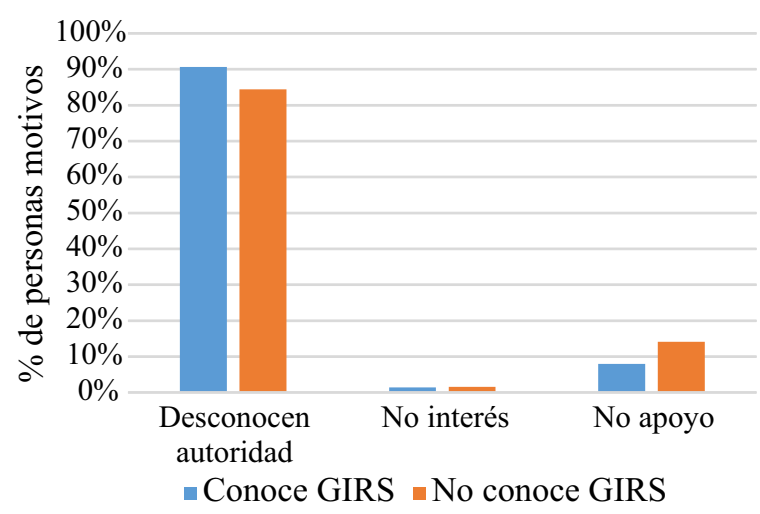

Figura 6. Disposición final de los residuos sólidos.

\section{IV.DISCUSIÓN}

La escasa educación ambiental y participación de la población en talleres de capacitación y sensibilización sobre el manejo de residuos sólidos tuvo repercusión en la inadecuada gestión integral de residuos sólidos. Esto se corroboró con el estudio realizado por SGAB (2008), que en su investigación mencionaron que el factor que había contribuido en la mala costumbre y tolerancia a la mala gestión de residuos del ciudadano cochabambino, fue la escasa educación y una capacitación reducida.

Tumi (2014), de igual manera, analizó la participación ciudadana en la gestión de residuos sólidos urbanos. Los resultados denotaron que los procesos de participación ciudadana tienen el potencial de transformar la gestión de residuos sólidos hacia un manejo integral y sustentable con la condición de incorporar a la ciudadanía en diversos procesos.

Factores socioeconómicos como los escasos recursos económicos de los gobiernos locales, la administración de los mismos para el mejoramiento de los servicios que brindaba, la falta de la participación ciudadana en los distintos procesos que involucraron a la gestión, y asociados a ellas los hábitos de costumbre y el entorno en donde se encuentran, son cuatro de las principales dificultades en la gestión integral de los residuos sólidos. Esto lo corroboró Chávez (2002), quien examinó las ventajas y limitaciones de las condiciones institucionales en las municipalidades de Perú, específicamente en lo referente a la gestión ambiental, con el objetivo de proponer recomendaciones y alternativas jurídico-institucionales que facilitaran tal gestión. Esta concluye diciendo que las iniciativas municipales no cuentan con una sólida participación de organizaciones comunales y que la relación con la sociedad civil es débil. Además existen y tienen una repercusión considerable la duplicidad de funciones y actividades con otras instituciones. Los gobiernos municipales cuentan con recursos económicos insuficientes y poca efectividad en su accionar, lo que ha provocado el desgaste y la deslegitimación como gobierno local, entre otras razones.

Sánchez (2007), dio a conocer que en cada entorno existen posibilidades y limitaciones distintas, por ello es importante tener una visión ordenada y compleja de las alternativas y que de acuerdo a las características socioeconómicas y culturales de la zona de estudio se gestione un manejo integral de los propios residuos sólidos.

Jaramillo (1999), menciona que la gestión de residuos sólidos es una tarea muy compleja que se ha convertido en un problema común en los países en vías de desarrollo, debido a múltiples factores, como el incremento de la población, la cantidad cada vez mayor de residuos que genera la población, la crisis económica que ha obligado a reducir el gasto público y a mantener tarifas bajas en detrimento de la calidad del servicio de aseo urbano, la debilidad institucional y la poca educación sanitaria y participación ciudadana.

De hecho, las referencias consultadas nos muestran cierta similitud con la investigación realizada, debido a que varios de los autores descritos en los párrafos anteriores nos dan a conocer que los factores sociales, económicos y políticos tienen gran repercusión en la gestión integral de los residuos sólidos. 


\section{CONCLUSIONES}

En la evaluación y análisis realizado en la gestión integral de residuos sólidos en el distrito de María, se encontró que el $60 \%$ de la población conoce la gestión integral de residuos sólidos, mientras que el $40 \%$ la desconoce.

Dentro de los principales problemas en la gestión integral de residuos sólidos en el distrito de María, se encontró la escasa educación ambiental y participación ciudadana en los diferentes servicios que brindó la municipalidad tales como barrido, recolección y transporte, debido a la falta de eficiencia y eficacia de los servicios.

Por último, los factores socioeconómicos que influyeron en la inadecuada GIRS se determinaron en base a la edad, género, rol que cumple en la familia, participación en capacitaciones sobre residuos sólidos, grado de instrucción, integrantes de la familia, falta de participación ciudadana, escasa información, ingreso promedio de la familia, escasos recursos económicos con los que cuenta la municipalidad (en este caso de María).

\section{RECOMENDACIONES}

Con el fin de mejorar la Gestión Integral de Residuos Sólidos (GIRS), se ha propuesto implementar el Plan de Manejo de Residuos Sólidos (PMRS) aprobado mediante ordenanza $\mathrm{N}^{\circ}$ 04-2015 MDM-A del distrito de María, el cual involucra las siguientes líneas de acción:

- Fortalecer la gestión municipal en cuanto a su capacidad técnica, operativa, gerencial, legal y financiera para asegurar la adecuada prestación integral del servicio.

- Fortalecer la institucionalidad municipal a través de la consolidación de la participación interinstitucional y la afinidad de esfuerzos en la gestión de los residuos sólidos, que garantice la continuidad y sostenibilidad del proceso de implementación.

- Incrementar los niveles de sensibilización ambiental en la población y los diferentes grupos de interés organizados en el distrito en temas de gestión ambiental, especialmente en gestión de residuos sólidos incluyendo a los que toman las decisiones, con énfasis en la promoción de la cultura de pago.

Otras acciones a apoyar son elaborar e implementar el programa de segregación en la fuente teniendo en cuenta que gran parte de la población separa sus residuos, promover una cultura de pago en la población por los diferentes servicios que brinda la municipalidad en función de la eficiencia y eficacia de los mismos, y finalmente contar con una adecuada disposición final de residuos sólidos, clausurando y promoviendo la restauración del botadero actual.

\section{REFERENCIAS BIBLIOGRÁFICAS}

Chávez, S. "Condiciones institucionales y jurídicas para la gestión ambiental de los gobiernos locales en Costa Rica”. Universidad de Costa Rica. San José (Costa Rica), 2002.

Jaramillo, J. "Seminario internacional: Gestión integral de residuos sólidos y peligrosos, siglo XXI". Universidad de Antoquia. Medellín (Colombia), 1999.

Martínez, N.M.J. "La gestión integral de residuos sólidos urbanos en México: entre la intención y la realidad. Letras Verdes". Revista Latinoamericana de Estudios Socioambientales, 17(2015): 29-56.

Postand y Baund. "Solid waste management and recycling; actors, partnerships and policies in Hyderabad, India and Nairobi, Kenya". USA, 2004.

Sánchez, G. "Gestión integral de residuos sólidos urbanos en municipios de Actopan, San Salvador y Arenal del Estado de Hidalgo. San Salvador (E1 Salvador), 2007.

SGAB (Sociedad de Gestión Ambiental Boliviana). "Cochabamba: Equipo ciudades focales. Conocimiento, educación y participación de la población de Cochabamba en el manejo de los residuos sólidos”. Cochabamba (Bolivia), 
2008. Recuperado de: http://cdam.minam. gob.pe/publielectro/investigacion $\% 20 \mathrm{amb}$

iental/investigacioneducacionambiental1.pdf

Tumi, J. “Representaciones sociales de la población de la ciudad de Puno sobre gestión de residuos sólidos". Revista de investigaciones alto andinas, 16(2014): 59-74. 